EPJ Web of Conferences 113,06011 (2016)

DOI: $10.1051 /$ epjconf/201611306011

(C) Owned by the authors, published by EDP Sciences, 2016

\title{
Exploring the Structure of the Most Neutron-rich Boron and Carbon Isotopes
}

\author{
N.A.Orr ${ }^{1, a}$ \\ ${ }^{1}$ LPC-Caen, ENSICAEN, Université de Caen Normandie, CNRS/IN2P3, 14050 Caen cedex, France
}

\begin{abstract}
The most neutron-rich isotopes of boron and carbon are of considerable interest in terms of their few-body character - most notably through the possible formation of twoneutron haloes in the Borromean nuclei ${ }^{19} \mathrm{~B}$ and ${ }^{22} \mathrm{C}$. Here a series of recent investigations are briefly reviewed, including preliminary results from reaction studies of ${ }^{18} \mathrm{~B}$ and ${ }^{21} \mathrm{C}$. It is concluded that ${ }^{22} \mathrm{C}$ exhibits a prominent halo arising from a significant $v 2 s_{1 / 2}^{2}$ valence neutron configuration. More generally, the structure of the dripline boron and carbon nuclei appears to be governed by the competition between the $v 1 d_{5 / 2}$ and $v 2 s_{1 / 2}$ singleparticle orbitals, with a $5 / 2^{+}-1 / 2^{+}$level inversion very probably occuring in ${ }^{21} \mathrm{C}$.
\end{abstract}

\section{Introduction}

The investigation of the light neutron-rich dripline nuclei, including in particular those exhibiting a halo, is a central theme of nuclear structure physics. In recent years advances have been made through the development of increasingly more sophisticated models, such as ab intio approaches and those incorporating explicitly the effects of the continuum. Experimentally, with the advent of ever more powerful facilities - most recently, the RIKEN-RIBF - detailed studies have become possible for nuclei lying at and beyond the driplines for $\mathrm{Z}>4$.

In this context, of particular interest are the heaviest candidate two-neutron halo systems, ${ }^{19} \mathrm{~B}$ and ${ }^{22} \mathrm{C}$ and the associated unbound sub-systems ${ }^{18} \mathrm{~B}$ and ${ }^{21} \mathrm{C}$, the continuum states of which are critical to the defining the ${ }^{17} \mathrm{~B}-\mathrm{n}$ and ${ }^{20} \mathrm{C}-\mathrm{n}$ interactions for three-body models. In addition, ${ }^{18,19} \mathrm{~B}$ and ${ }^{21,22} \mathrm{C}$ are of considerable interest in terms of the evolution of shell-structure far from stability as they span the $\mathrm{N}=14$ and 16 sub-shell closures below doubly-magic ${ }^{22,24} \mathrm{O}$. Indeed, the level structures of ${ }^{18} \mathrm{~B}$ and ${ }^{21} \mathrm{C}$ can shed light on the evolution of the $v 2 s_{1 / 2}$ and $v 1 d_{5 / 2}$ levels which may become degenerate in this region [1].

In terms of ascertaining the halo character of a nucleus, measurements of total reaction cross sections (ideally across a chain of isotopes) provide the first indications [2]. In practice, such measurements may be carried out at very low beam intensities, a considerable advantage at the dripline. In the case of ${ }^{19} \mathrm{~B}$ measurements were undertaken some time ago at very high energy ( $800 \mathrm{MeV} /$ nucleon) and interpreted in terms of a Glauber optical model analysis [3]. An RMS matter radius of $3.11 \pm 0.13 \mathrm{fm}$ was deduced for ${ }^{19} \mathrm{~B}$ as compared to $2.99 \pm 0.09 \mathrm{fm}$ for ${ }^{17} \mathrm{~B}$. As such ${ }^{19} \mathrm{~B}$ did not appear to exhibit a strongly developed two-neutron halo, but rather, through comparison with the less neutron rich boron isotopes, it was suggested that it may be better described as a ${ }^{15} \mathrm{~B}$ core and 4 valence neutrons.

\footnotetext{
a e-mail: orr@1pccaen.in2p3.fr
} 


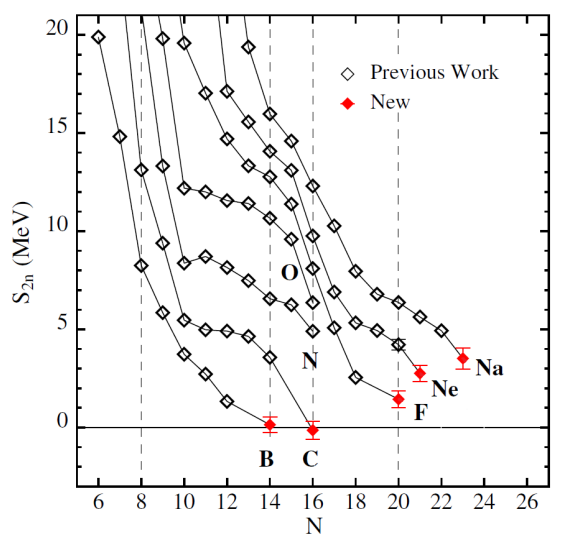

Figure 1: Two-neutron separation energies derived from experimentally determined masses. The $\mathrm{N}=8$, 14, 16 and 20 (sub-) shell closures are indicated. Adapted from Ref. [6].

In the case of ${ }^{22} \mathrm{C}$ intermediate energy ( $40 \mathrm{MeV} /$ nucleon) measurements undertaken more recently found a very large enhancement of the total reaction cross section with respect to the less neutron-rich neighbouring isotopes [4]. Using a similar Glauber optical limit approach an RMS matter radius of $5.4 \pm 0.9 \mathrm{fm}$ was deduced - very much larger than systematics would suggest and very much larger than the well-established single-neutron halo ${ }^{19} \mathrm{C}$. It was concluded that such a large matter radius may be attributed to a strong $s$-wave valence neutron configuration.

\section{Masses and valence neutron binding energies}

One of the key ingredients in characterising halo systems is the valence neutron binding energy. Determining experimentally the masses of boron and carbon isotopes at the neutron dripline is not possible at present, however, using high precision techniques such as those employing traps. Indeed, current ISOL based techniques are unable to provide even the weakest of beams of such nuclei. As such, in-flight fragmentation followed by a direct time-of-flight and rigidity measurement represents the only viable approach [5]. Such measurements have been made at GANIL with a ${ }^{48} \mathrm{Ca}$ beam and the SISSI+alpha spectrometer facility coupled to the SPEG spectrometer [6]. Using well established masses as references [7], the mass excesses of 16 light neutron-rich nuclei were determined, including those of ${ }^{19} \mathrm{~B}$ and ${ }^{22} \mathrm{C}$ for the first time ${ }^{1}$.

The two-neutron separation energies $\left(S_{2 n}\right)$ derived from the measured masses and previously established values [7] are reported in Fig. 1 [6]. It is clear that ${ }^{19} \mathrm{~B}$ and ${ }^{22} \mathrm{C}$ lie at the very limits of binding $-S_{2 n}=0.14 \pm 0.39$ and $-0.14 \pm 0.46 \mathrm{MeV}$ respectively ${ }^{2}$ - suggesting that prominent neutron haloes may occur. These binding energies may be employed, in conjunction with the radii deduced from the interaction cross section measurments, to provide a first insight into the valence neutron configurations.

In a simple picture [9], the matter radius, $R_{m}$, may be estimated as,

$$
R_{m}^{2}=\frac{A-k}{A}\left(R_{c}^{2}+\frac{k}{A} r_{v}^{2}\right)
$$

\footnotetext{
${ }^{1}$ The first mass measurements were also made for ${ }^{29} \mathrm{~F}$ and ${ }^{34} \mathrm{Na}$ together with the most precise determinations for ${ }^{23} \mathrm{~N}$ and the deformed, single-neutron halo system ${ }^{31} \mathrm{Ne}$ [8].

${ }^{2}$ Given that ${ }^{22} \mathrm{C}$ is bound, this may be taken as providing an upper limit $(1 \sigma)$ for the $S_{2 n}$ of $0.32 \mathrm{MeV}$.
} 

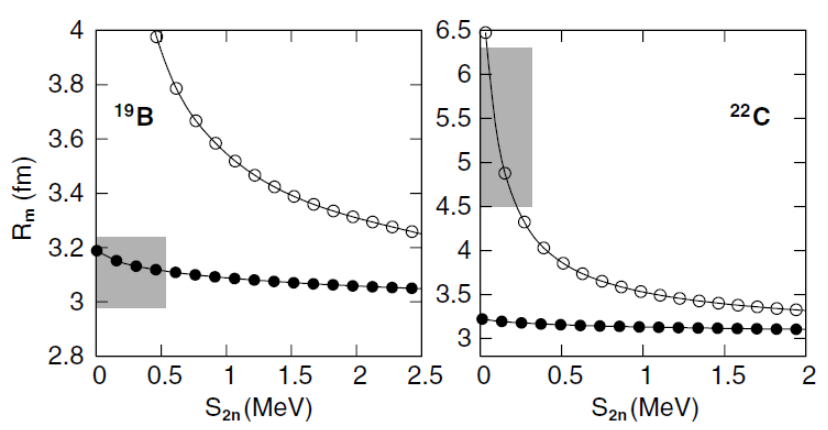

Figure 2: Matter radii $\left(R_{m}\right)$ for ${ }^{19} \mathrm{~B}$ and ${ }^{22} \mathrm{C}$ as a function of the two neutron binding energy $\left(S_{2 n}\right)$. Curves marked with symbols correspond to calculations assuming different valence neutron configurations: $v 2 s_{1 / 2}^{2}$ open circles and $v 1 d_{5 / 2}^{2}$ filled points. The experimentally deduced $R_{m}[3,4]$ and mass determinations [6] are shown (shaded rectangles). Adapted from Ref. [6].

where $A$ is the mass number of the nucleus, $k$ the number of valence neutrons, $R_{c}$ the matter radius of the core nucleus, and $r_{v}$ the radius of the valence neutron distribution. The $R_{c}$ adopted were: $2.99 \pm 0.09 \mathrm{fm}$ for ${ }^{17} \mathrm{~B}[3]$ and $2.98 \pm 0.05 \mathrm{fm}$ for ${ }^{20} \mathrm{C}[10]$. The valence neutron radius may be estimated using a Woods-Saxon potential $\left(a=0.65 \mathrm{fm}\right.$ and $\left.r_{0}=1.25 \mathrm{fm}\right)$, where the depth is adjusted to ensure the correct binding of each valence neutron $(n, \ell)$ where it is assumed that the the binding energy $\left(\mathrm{S}_{2 n}\right)$ is shared equally between them. The results for a range of valence neutron binding energies are displayed for both the $s$ and $d$-wave neutron configurations in Fig. 2 along with the experimentally determined $R_{m}$ and $S_{2 n}$, where the matter radii for ${ }^{19} \mathrm{~B}$ and ${ }^{22} \mathrm{C}$ noted in the preceding section were employed. As may be seen, the results for ${ }^{19} \mathrm{~B}$ are well reproduced when the valence neutrons are assumed to be predominantly of $d$-wave character. This suggests that the radial extent of the valence neutron distribution will be significantly suppressed by the angular momentum barrier. On the contrary, the results for ${ }^{22} \mathrm{C}$ strongly support the devopment of a two-neutron halo with a predominately $s$-wave configuration in line with the conclusion of the total reaction cross section study [4] (Section 1).

\section{Momentum Distributions}

As was first demonstrated in some of the earliest studies of halo nuclei, the momentum distribution of the breakup prodcuts - in particular the core - can provide a clear signature of their halo character through their narrow widths $[11,12]$. More recently, the momentum distributions from reactions on a low-Z target (such as Be and $\mathrm{C}$ ) and associated cross sections have been developed as a spectroscopic tool [13, 14], as demonstrated, for example, by the "text book" case of the systematic study of Ref. [15]. In simple terms, the core-like reaction residue, following high-energy neutron removal (or "knockout") carries rather directly the imprint of the orbital angular momentum of the valence neutron. On the other hand, the cross section, when compared with reaction calculations (typically within the eikonal approach), provides a measurement of the spectroscopic strength between the initial (projectile ground-state) and final (core residue) state [14].

In the case of ${ }^{22} \mathrm{C}$, the secondary beam intensities available during the early stage operations of the RIKEN-RIBF enabled inclusive measurements to be made of two-neutron removal on a carbon target at some $240 \mathrm{MeV} /$ nucleon [16]. For comparison, measurements were also made on the neighbouring carbon isotopes, including ${ }^{20} \mathrm{C}$. The momentum distributions were derived using a time-of-flight 

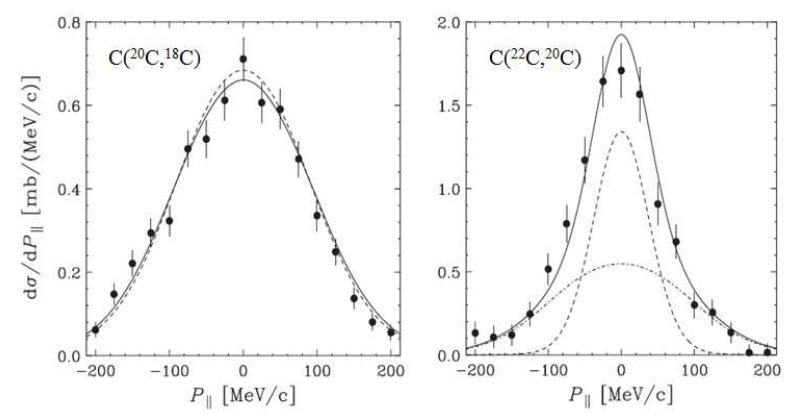

Figure 3: Inclusive longitudinal momentum distribution for two-neutron removal from ${ }^{20} \mathrm{C}$ and ${ }^{22} \mathrm{C}$. The results of eikonal reaction theory calculations are shown (solid lines). In the case of ${ }^{22} \mathrm{C}$, the components arising from $s$-wave (dashed) and $d$-wave (dot-dashed line) neutron removal are also shown. Adapted from Ref. [16].

technique and a spectrometer operating at zero-degrees. The results are shown in Fig. 3 whereby the narrowness of the momentum distribution for two-neutron removal from ${ }^{22} \mathrm{C}$, as compared to ${ }^{20} \mathrm{C}$, is clear.

In the case of a Borromen nucleus such as ${ }^{22} \mathrm{C}$, essentially all the yield is produced via singleneutron removal followed by in-flight decay of the unbound ${ }^{21} \mathrm{C}$ [17]. As discussed in detail in Ref. [16], combining shell model predictions obtained using the WBP interaction [18], within the psd model space, with eikonal reaction calculations (including the recoil from the decay of the ${ }^{21} \mathrm{C}$ states) the ${ }^{20} \mathrm{C}$ core momentum distribution was very well reproduced, in both form and magnitude. As expected, the narrowness of the distribution arises from the $s$-wave removal component. This result not only confirms the importance of the $v 2 s_{1 / 2}^{2}$ valence neutron configuration but also suggests that ${ }^{21} \mathrm{C}$ has low-lying $1 / 2^{+}$and $5 / 2^{+}$levels. In the case of ${ }^{19} \mathrm{~B}$, while no measurements are yet available of the core-residue momentum distribution, the shell model calculations suggest that closely spaced levels ${ }^{3}$ arising from the $v 2 s_{1 / 2}$ and $v 1 d_{5 / 2}$ configurations dominate the low-lying level scheme of ${ }^{18} \mathrm{~B}$ [19].

\section{Invariant mass spectroscopy of ${ }^{18} \mathrm{~B}$ and ${ }^{21} \mathrm{C}$}

In order to investigate directly the low-lying level structures of ${ }^{18} \mathrm{~B}$ and ${ }^{21} \mathrm{C}$, the technique of invariant mass spectroscopy following in-flight decay was employed. In addition, the complementary probes of neutron-removal from ${ }^{19} \mathrm{~B}$ and ${ }^{22} \mathrm{C}$, together with single-proton removal from ${ }^{19} \mathrm{C}$ and ${ }^{22} \mathrm{~N}$, were used to populate different final-states of ${ }^{18} \mathrm{~B}$ and ${ }^{21} \mathrm{C}$. In simple terms, proton removal is expected to populate final states with the same neutron configurations as the projectile ( $v 2 s_{1 / 2}$ in both cases), whilst neutron removal will probe directly the valence neutron configurations (here, most probably, $v 2 s_{1 / 2}^{2}$ and $v 1 d_{5 / 2}^{2}$ ). As for the momentum distribution study, the secondary beams were generated by the RIKEN-RIBF facility. The measurements were performed using the SAMURAI spectrometer [20] coupled to the large area neutron array NEBULA [21].

The preliminary results for the reconstructed fragment-neutron relative energy $\left(\mathrm{E}_{r e l}\right)$ spectra - that is the energy above the single-neutron emission threshold - are displayed in Figs 4 and 5. In the case of proton removal from ${ }^{19} \mathrm{C}$, the ${ }^{17} \mathrm{~B}+\mathrm{n}$ spectrum exhibits a very strong accumulation of strength at

\footnotetext{
${ }^{3}$ Multiplets, owing to the coupling to the $\pi 1 p_{3 / 2}$ hole.
} 

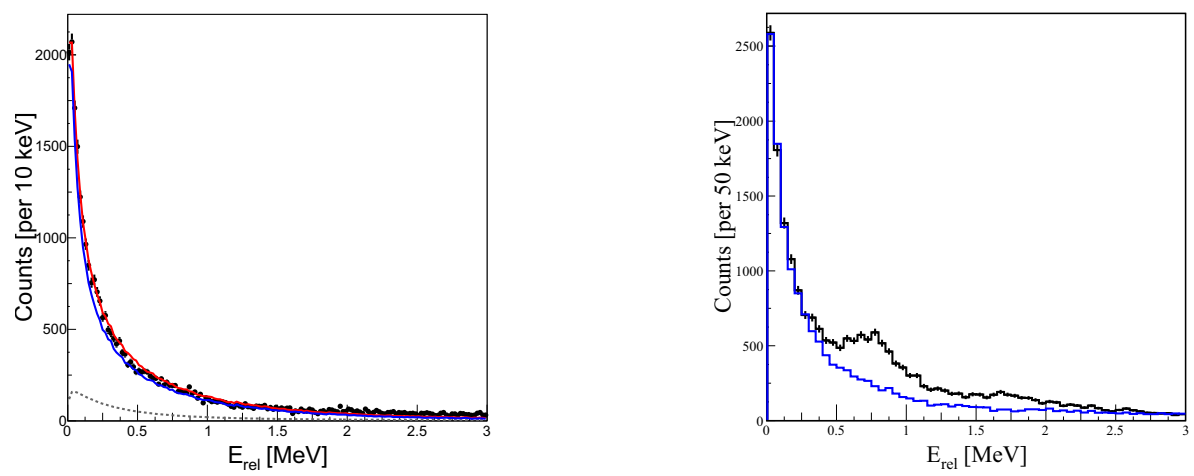

Figure 4: (Colour online) Relative energy spectra, as shown by the data points, for the $\mathrm{C}\left({ }^{19} \mathrm{C},{ }^{17} \mathrm{~B}+\mathrm{n}\right)$ (left) and $\mathrm{C}\left({ }^{19} \mathrm{~B},{ }^{17} \mathrm{~B}+\mathrm{n}\right)$ (right panel) reactions. In the former a fit (red) is shown including a strongly interacting $s$-wave virtual state (blue) and a small non-resonant continuum contribution (dotted line). In the latter, comparison is shown with the spectrum, renormalised at low $\mathrm{E}_{r e l}$, for the $\mathrm{C}\left({ }^{19} \mathrm{C},{ }^{17} \mathrm{~B}+\mathrm{n}\right)$ reaction (blue histogramme).

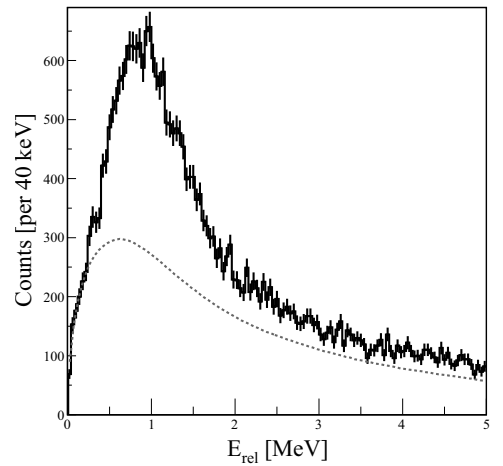

(a) $\mathrm{C}\left({ }^{22} \mathrm{~N},{ }^{20} \mathrm{C}+\mathrm{n}\right)$

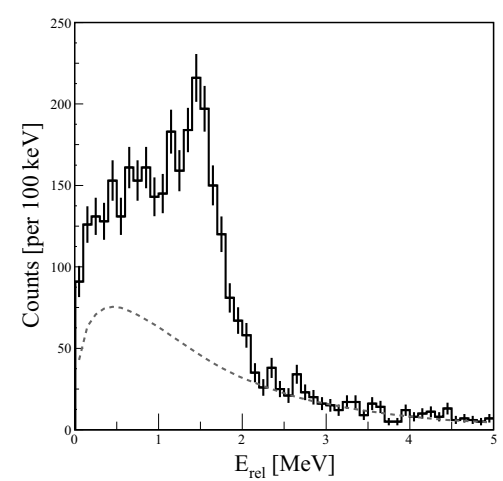

(b) $\mathrm{C}\left({ }^{22} \mathrm{C},{ }^{20} \mathrm{C}+\mathrm{n}\right)$

Figure 5: Relative energy spectra for the $\mathrm{C}\left({ }^{22} \mathrm{~N},{ }^{20} \mathrm{C}+\mathrm{n}\right)($ left $)$ and $\mathrm{C}\left({ }^{22} \mathrm{C},{ }^{20} \mathrm{C}+\mathrm{n}\right)$ (right panel) reactions. Comparison is shown with non-resonant continuum distributions (dotted lines).

threshold. Such behaviour is indicative of a strongly interacting virtual $s$-wave state [22] and indeed the spectrum, as shown, may be very well described with such a state with a scattering length of less than $-50 \mathrm{fm}$ - in agreement with an earlier study [19] - and a small contribution from the non-resonant continuum [23]. In the case of neutron removal from ${ }^{19} \mathrm{~B}$, in addition to the strong $s$-wave strength a broad resonance-like structure is present at around $0.8 \mathrm{MeV}$ with a possible second weaker structure at $\sim 1.7 \mathrm{MeV}$. The former lies close to the predicted location of the $2^{-}-4^{-}$levels arising primarily from the $1 d_{5 / 2}$ valence neutron [19].

Turning to the results for ${ }^{21} \mathrm{C}$ (Fig. 5), the relative energy spectrum for proton removal from ${ }^{22} \mathrm{~N}$ is, in contrast to an earlier very low statistics study [24], dominated by a prominent resonance-like structure at around $1 \mathrm{MeV}$ which may be identified, based on the selectivity of the reaction and width, 
as an $s$-wave resonance. In the case of the neutron removal from ${ }^{22} \mathrm{C}$, a relatively narrow structure is observed close to $1.5 \mathrm{MeV}$ above threshold, together with a broad distribution of lower-lying strength. A first examination of the reconstructed ${ }^{21} \mathrm{C}$ momentum distribution indicates that the narrow structure may be identified with $d$-wave neutron removal, whilst the low-lying strength is consistent with $s$-wave neutron removal. Further analysis will be required to confirm these propositions, however it may be noted that they are in line with the shell-model predictions for the ${ }^{21} \mathrm{C}$ level scheme, which predict an inversion of the lowest lying $5 / 2^{+}$and $1 / 2^{+}$states [16].

In addition to refining the analyses and interpretation, including examining ${ }^{17} \mathrm{~B}$ and ${ }^{20} \mathrm{C}-\gamma-n$ coincidences, data acquired for dissociation of both the ${ }^{19} \mathrm{~B}$ and ${ }^{22} \mathrm{C}$ beams on a lead target is also being analysed with the goal of extracting the $\mathrm{E} 1$ strength functions. The latter should provide further insight into the structure and halo character of both nuclei.

\section{Acknowledgements}

I am grateful to all my collaborators in the studies described here, including, in particular, Nobuyuki Kobayashi, Takashi Nakamura and Jeff Tostevin (momentum distributions), Laurent Gaudefroy and Wolfi Mittig (mass measurments), Sylvain Leblond, Miguel Marqués and Julien Gibelin (spectroscopy of ${ }^{18} \mathrm{~B}$ and ${ }^{21} \mathrm{C}$ ). Support from the Franco-Japanese LIA-International Associated Laboratory for Nuclear Structure Problems is also acknowledged.

\section{References}

[1] G. Jansen et al., Phys. Rev. Lett. 113, 142502 (2014)

[2] I. Tanihata et al., Phys. Rev. Lett. 55, 2676 (1985).

[3] T. Suzuki et al., Nucl. Phys. A 658, 313 (1999).

[4] K. Tanaka et al., Phys. Rev. Lett. 104, 062701 (2010).

[5] W. Mittig, A. Lépine-Szily, N.A. Orr, Ann. Rev. Nucl. Part. Sci. 47, 27 (1997).

[6] L. Gaudefroy et al., Phys. Rev. Lett. 109, 20503 (2012).

[7] W. Meng et al., Chin. Phys. C 36, 1603 (2012).

[8] T. Nakamura et al., Phys. Rev. Lett. 103, 262501 (2009).

[9] P.G. Hansen, B. Jonson, Europhys. Lett. 4, 409 (1987).

[10] A. Ozawa et al., Nucl. Phys. A 691, 599 (2001).

[11] T. Kobayashi et al., Phys. Rev. Lett. 60, 2599 (1988).

[12] N.A. Orr et al., Phys. Rev. Lett. 69, 2050 (1992).

[13] P.G. Hansen, J.A. Tostevin, Ann. Rev. Nucl. Part. Sci. 53, 219 (2003).

[14] J.A. Tostevin, J. Phys. G 25, 735 (1999).

[15] E. Sauvan et al., Phys. Lett. B 491, 1 (2000).

[16] N. Kobayashi et al., Phys. Rev. C83, 054604 (2012).

[17] F. Barranco et al., Phys. Lett. B 319, 387 (1993).

[18] E.K. Warburton and B.A. Brown, Phys. Rev. C 46, 923 (1992).

[19] A. Spyrou et al., Phys. Lett. B 683, 129 (2010).

[20] T. Kobayashi et al., Nucl. Instr. Meth. B 317, 294 (2013).

[21] Y. Kondo et al., RIKEN Accel. Prog. Rep. 45, 131 (2012);

[22] M. Thoennessen et al., Phys. Rev. Lett. 59, 111 (1999).

[23] G. Randisi, Phys. Rev. C 89, 034320 (2014).

[24] S. Mosby et al., Nucl. Phys. A 909, 69 (2013). 